\title{
Integrated Monitoring of Encoder Status Parameters and GUI based Remote Control Panel Using Lab view
}

\author{
Amiya Ranjan Panda \\ Junior Research Fellow \\ Integrated Test Range, \\ Defence R\&D Organization \\ Balasore, India
}

\author{
Utpal Mandal \\ Scientist \\ Integrated Test Range, \\ Defence R\&D Organization \\ Balasore, India
}

\author{
Hare Krishna Ratha \\ Scientist \\ Integrated Test Range, \\ Defence R\&D Organization \\ Balasore, India
}

\begin{abstract}
This paper describes the necessity of "Integrated Monitoring of Encoder status parameters and GUI based Remote Control Panel using LabVIEW" application, which is the implementation of serial and Ethernet data transmission using LabVIEW. Remote Control Panel (RCP) is an electronic instrument from which specified commands can be given to Encoders. The RCP is generally connected to the command Encoders through serial links (RS 232, RS 422). After getting the commands from RCP, the commands are generated at Encoders and are transmitted followed by different processing. In the hardware based RCP system, some faults are observed at RCP and the facility to monitor the Encoders status parameters at RCP is not present. To overcome these bottlenecks, a Personal Computer (PC) based integrated RCP system was visualized and developed based on LabVIEW. LabVIEW was used for better GUI interface and reduction of development time. This system takes Encoder status parameters from Encoder change over unit through Ethernet link and is serially connected to the Encoders to gives commands to it. Hence all the status parameters of Encoders are monitored as well as appropriate commands can be given to the Encoders through this application.
\end{abstract}

\section{General Terms}

Serial data transmission; Ethernet data transmission.

\section{Keywords}

LabVIEW; Serial data transmission; Ethernet data transmission

\section{INTRODUCTION}

\subsection{Command Transmission System}

Command Transmission System (CTS) System basically involves in generation and transmission of remote command signals to the airborne vehicle through RF link to execute some operation required in real time flight of the vehicle.

Function of CTS System: CTS system is used for termination of flight vehicle under test. Termination of test vehicle is mandatory if the vehicle deviates from its preset trajectory and takes different course due to unpredictable failures of onboard system. In such cases terminate command is transmitted from ground CTS system for termination of test vehicle.

\subsection{Requirements}

CTS system consists of major components like RCP, Encoder/Decoder, Signal generator, Transmitter, Auto change Over Unit. Sequences of commands are transmitted to the flight vehicle through RCP. RCP is connected to the command Encoder through serial links. In the current system the facility to monitor the Encoders status parameters at RCP is not present. More over the current system is cost effective. The developed system is a software implementation of hardware based Remote Control Panel. By placing this system at a remote location, commands can be given as well as Encoder status information can be monitored through this system.

\subsection{System Configuration}

The new integrated Remote Control Panel is connected to the dual chain CTS system through RS 232 and Ethernet links. The commands from the new RCP go to the Encoders through RS 232 link and the new integrated RCP takes the Encoder status information from Encoder Changeover Unit through UDP communication. After getting the command from RCP,

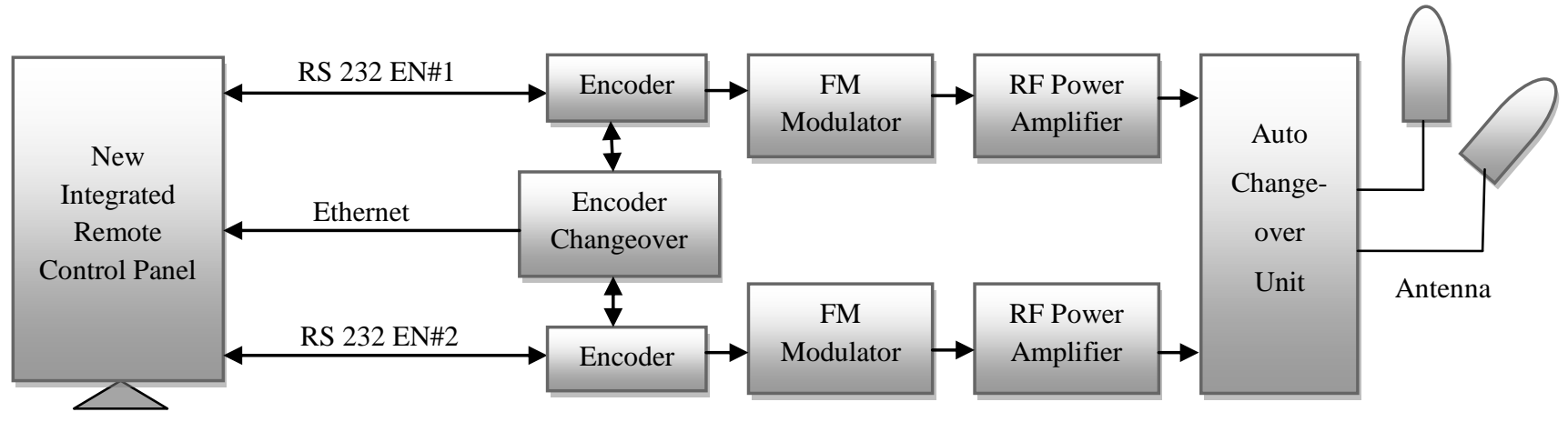

Figure 1. System Configuration 
the Encoder encodes it followed by modulation at FM modulator. Then the modulated signal is amplified at Power Amplifier and transmitted through Antenna.

\section{LabVIEW}

\subsection{LabVIEW Implementation}

LabVIEW is an imaginative graphical programming system which helps to develop computer-controlled data acquisition and analysis system [1]. Lab VIEW provides a perfect scope to accomplish computer-based research. The wired-together icons to perform simple-to-understand tasks of Lab VIEW make operations given below possible.
1. Building an array
2. Parsing a string
3. Digitalizing an analog voltage

Once a LabVIEW program is created and understood the modularity nature of it facilitates to include in the library that can be used later for more complicated program.

\subsection{LabVIEW Program Interface}

A LabVIEW program includes two windows, called the Front Panel and the Block Diagram.

\subsubsection{The Front Panel}

Once a program is developed, the front panel acts as the face of a laboratory instrument. Front panel includes controls and indicators, which are the interactive input and output terminals of the VI, respectively. Controls are knobs, push buttons, dials, and other input mechanisms. Indicators are graphs, LEDs, and other output displays. Hence the front panel is the program's user-interface, which facilitates the interaction of:

1. Providing inputs to the program,

2. Monitoring outputs from the program as it runs.

\subsubsection{The Block Diagram}

The actual LabVIEW programming code is exposed in the block diagram. It contains Lab View's well-stocked libraries of icons that we have selected during the program development. Each icon stands for a block of underlying executable code that does a particular useful function. The programming is done by wiring these icons properly, so that data flows amongst graphical images to achieve a desired function.

LabVIEW enables us to carry out state of the art research including:
1. Instrument Control
2. Data Acquisition
3. Data Analysis
4. Data Presentation and Data Storage.

\subsubsection{Virtual Instruments (VI)}

LabVIEW programs are called Virtual Instruments (VI) because their appearance and operation imitate physical instruments, such as oscilloscopes and multi meters [1]. Every VI uses functions that manipulate input from the user interface or other sources and display that information or more it to other files or other computers [2].

\section{SOFTWARE IMPLEMENTATION}

The developed application comprises of two units. 1. Data reception through Ethernet 2.Data transmission through serial port. In each of the above case data reception is followed by data processing and data presentation, which is shown in
Figure 2 and Figure 3. The commands from the RCP as well as the reception acknowledgement to the RCP are transmitted through serial port.
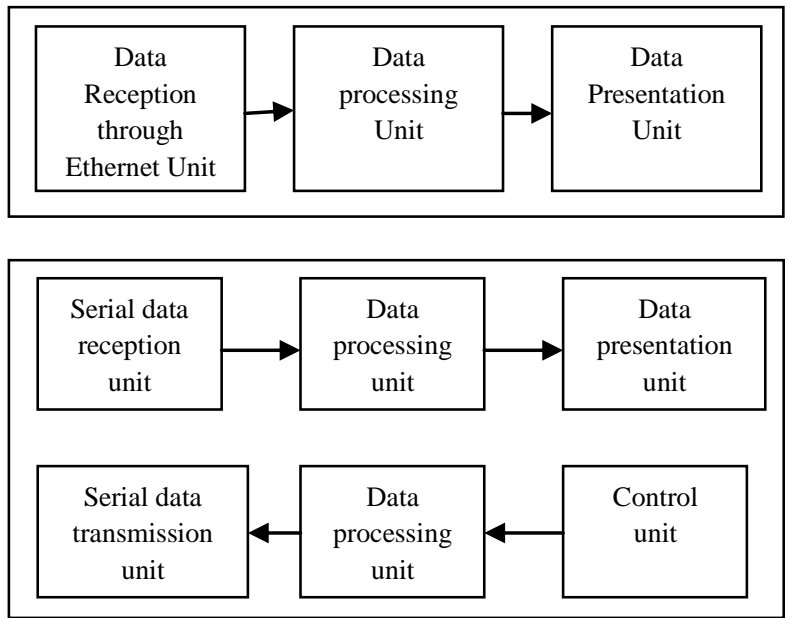

Figure 2. Dataflow in integrated Range Safety Panel

\subsection{Data reception through Ethernet}

First the UDP socket is opened then the data reception is done. After that buffer size is matched and then the control goes to the required unit accordingly.

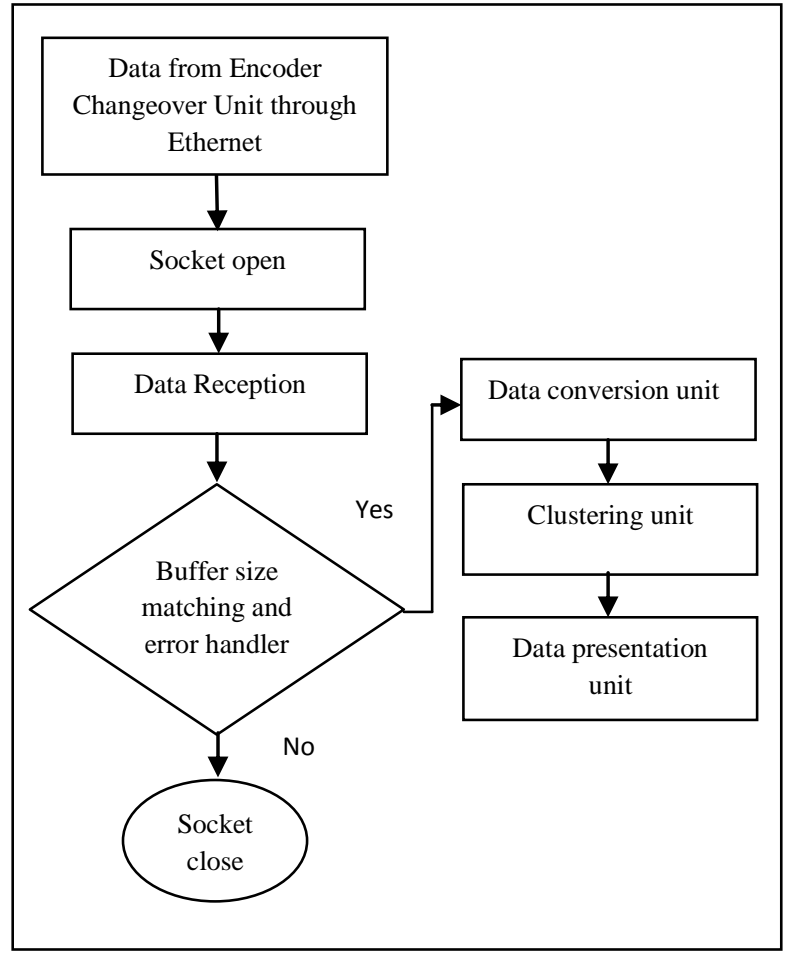

Figure 3. Flowchart of data reception through Ethernet

If the buffer size mismatch occurs then the socket is made close, otherwise control goes to conversion unit. The data received through UDP unit is clustered together and goes to the display unit. The above mentioned procedure is shown in Figure 3. 


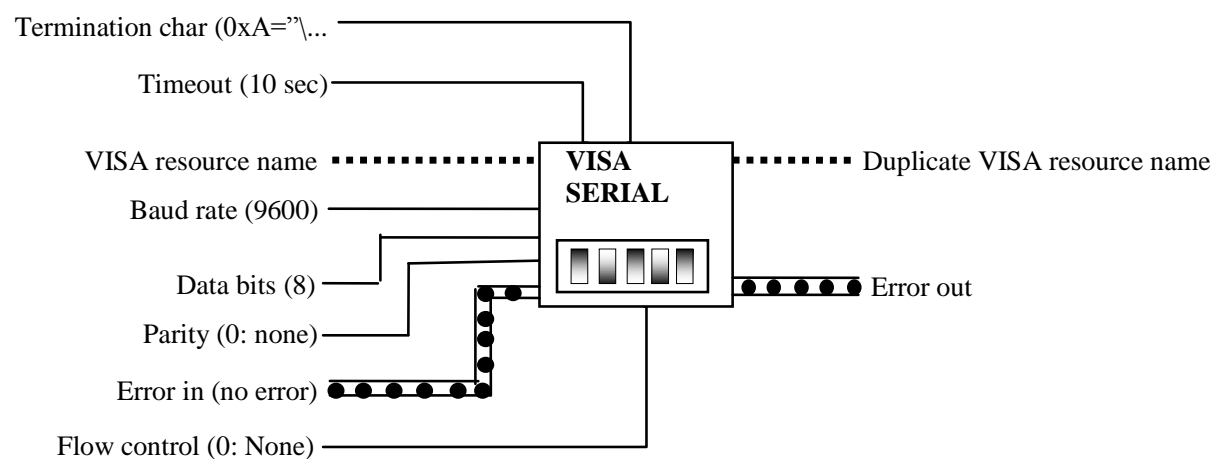

Figure 4. VISA configure serial port VI

\subsection{Data transmission through serial port}

Serial communication needs specification of four parameters: the baud rate of the transmission, the number of data bits encoding a character, the sense of the optional parity bit, and the number of stop bits. Each transmitted character is packaged in a character frame that consists of a single start bit followed by the data bits, the optional parity bit, and the stop bit or bits. Baud rate is a measure of how fast data is moving between instruments that use serial communication. RS-232 uses only two voltage states, called MARK and SPACE. In such a two state coding scheme, the baud rate is identical to the maximum number of bits of information, including "control" bits, which are transmitted per second. The start bit signifies the beginning of each character frame. It is a transition from negative (MARK) to positive (SPACE) voltage. In transmission of data bits, inverted logic is used and the order of transmission is from least significant bit (LSB) to most significant bit (MSB). An optional parity bit follows the data bits in character frame. The last part of the character frame is a stop bit. These bits are always represented by a negative voltage. If no characters are transmitted further, the line stays in negative voltage state. The transmission of next character frame is followed by a start bit of positive (SPACE) voltage [3].

The VISA Configure Serial Port VI initializes the port identified by VISA resource name to the specified settings. Timeout sets the timeout value for the serial communication. Baud rate, data bits, parity, and flow control specify those specific serial port parameters [4]. The VISA Configure Serial Port VI is shown in Figure 4.

In this application commands are given through GUI and transmitted through serial port interface. First the VISA configures serial port VI is initialized, where different serial port parameters are specified [5]. Then the serial port read and writes units are activated. Data from the control unit goes to the serial port write operation unit followed by conversion unit and then transmitted to Encoder unit. The data from the Encoder unit is received by serial read unit followed by conversion unit and string matching unit. Then the command information is displayed in data presentation unit. Figure 5 shows the above mentioned procedure [6].

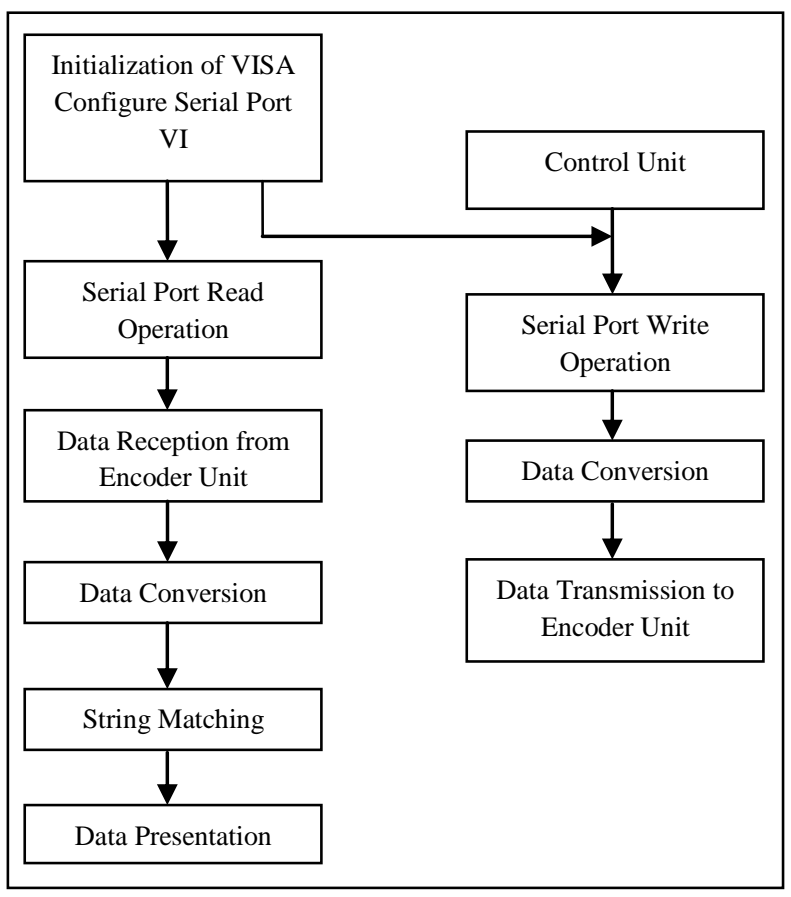

Figure 5. Flowchart of data transmission through serial port

\section{IMPORTANT FEATURES}

1. Commands can be given from remote location to the CTS system through this application. It is the software implementation of previous hardware based Remote Control Panel with some additional features.

2. Encoder's status parameters can be monitored at RCP through this system.

3. The development cost of this system is very less.

4. In future it can be modified according to the requirements without any extra cost. 


\section{RESULTS}

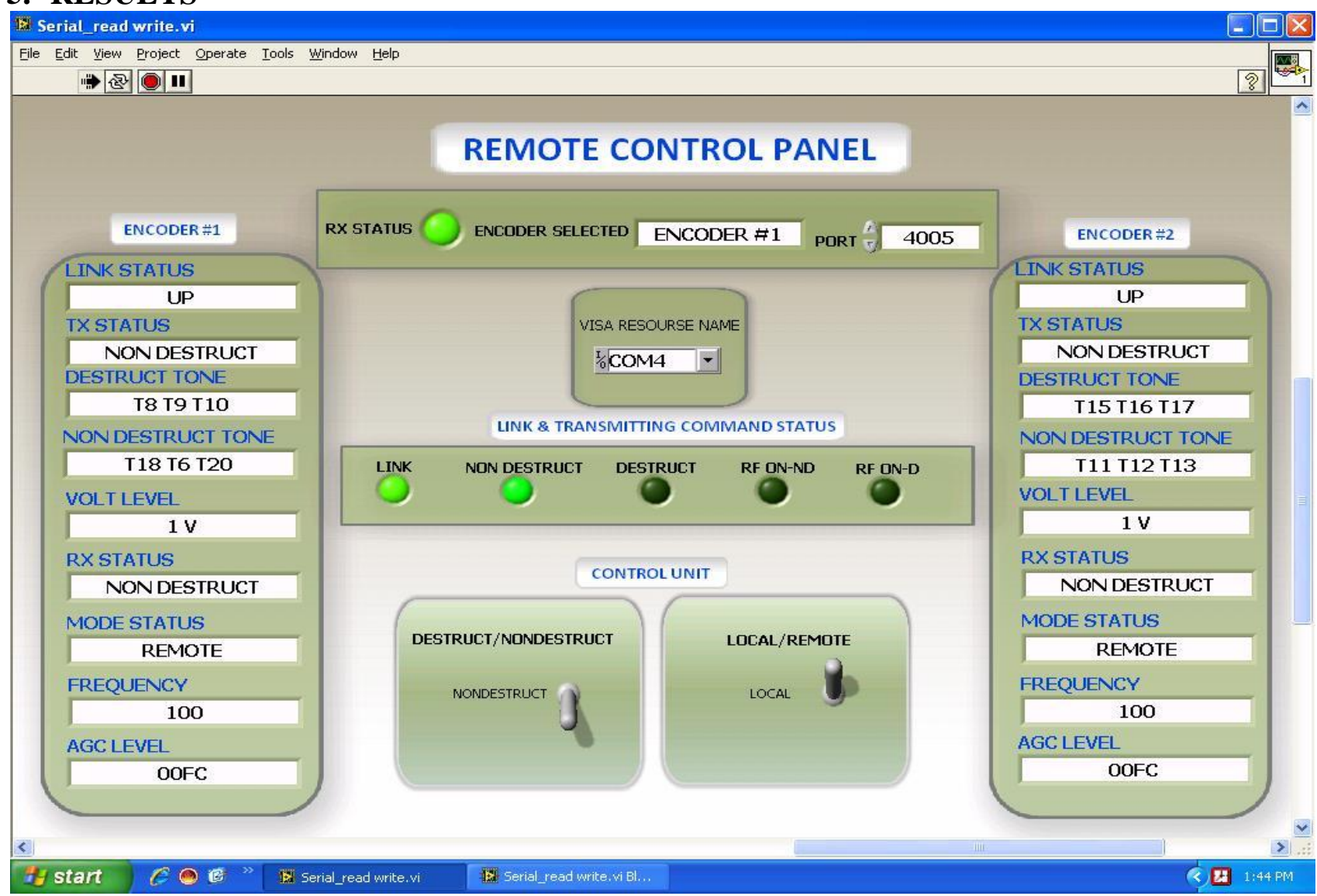

Figure 6. Integrated Remote Control Panel display and control unit

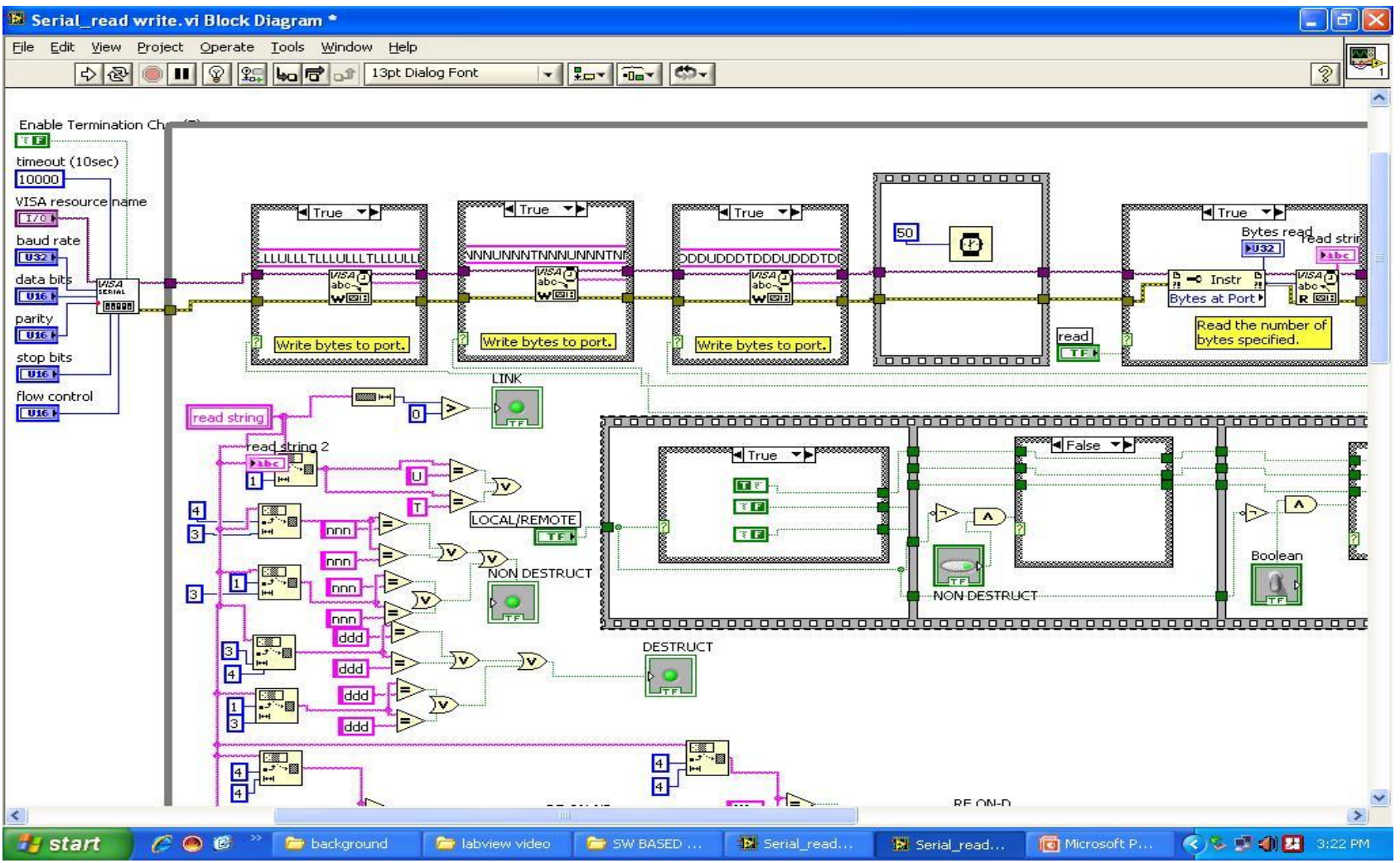

Figure 7. Block diagram showing serial read write 


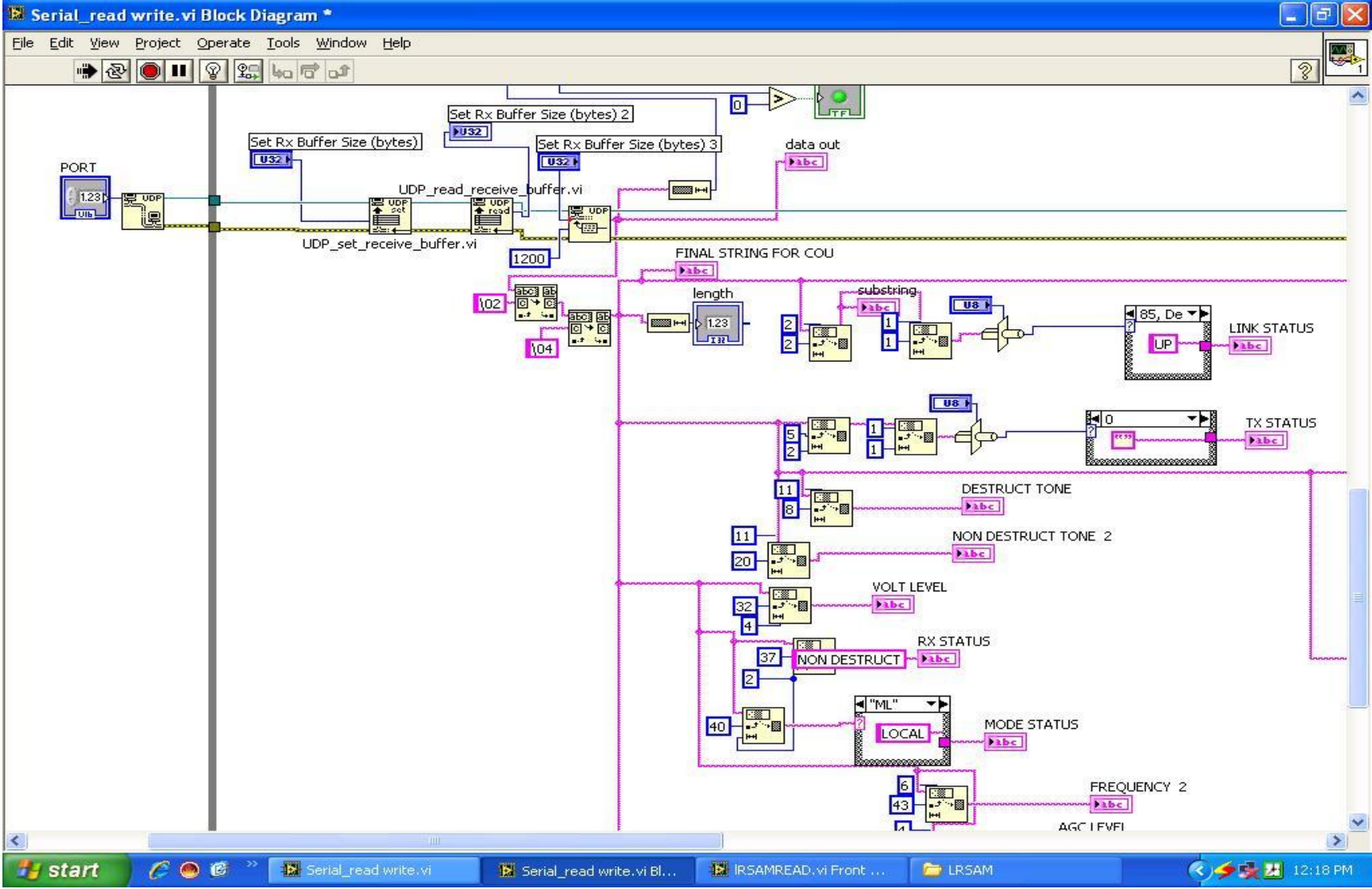

Figure 8. Block Diagram showing data reception through Ethernet

Figure 6 represents the integrated Remote Control Panel display and control unit. Through this window, commands can be given to Encoders and also the status parameters of Encoders can be monitored. This window has broken into different sub window for better description purpose. Figure 7 represents the code window showing serial read write unit [7]. Figure 8 shows the code window for data reception through Ethernet.

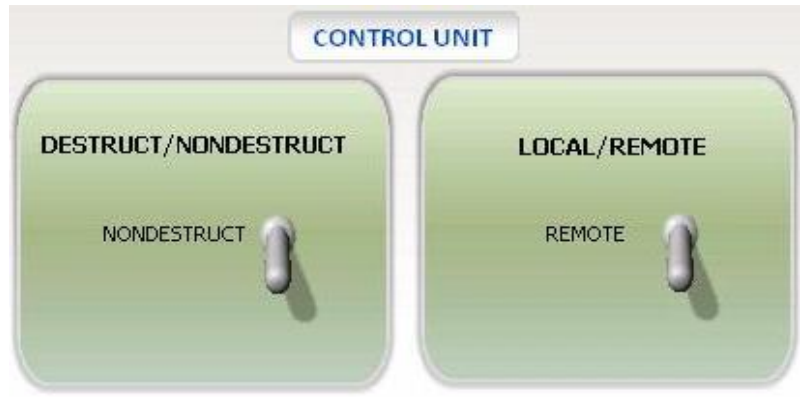

Figure 9. Displaying control unit

Figure 9 represents the control unit. Through this unit commands are given to the Encoders of the CTS system. This unit consists of two toggle switch sub unit. One unit is for mode selection i.e. Local/Remote and other one for command selection i.e. Nondestruct/Destruct. Local mode makes the CTS system to transmit commands from Encoder unit. Remote mode enables the Remote Control Panel unit to transmit commands [8].

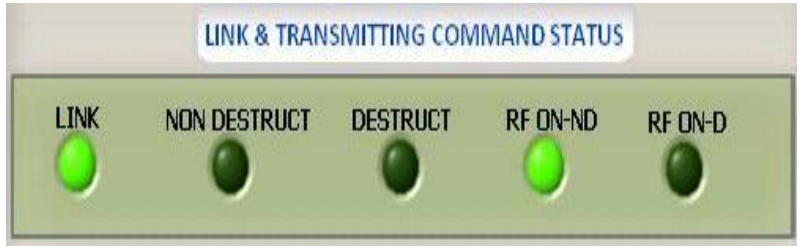

Figure 10. Serial link and transmitting command status

Figure 10 shows the serial link and transmitting command status display. It shows the presence and absence of RF and the transmitting command from RCP. LINK is an indicator which shows the serial link status. NONDESTRUCT, DESTRUCT shows the transmitting command status during RF off. RF ON-ND, RF ON-D shows the transmitting command status during RF on. After getting the acknowledgement from Encoder, all these indicators glow.

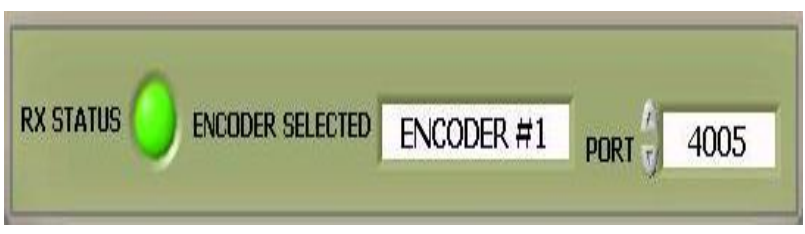

Figure 11. Displaying Ethernet link status, Encoder selection control and port number

Figure 11 displays the Ethernet link status, Encoder selection indicator and port number selection control. 


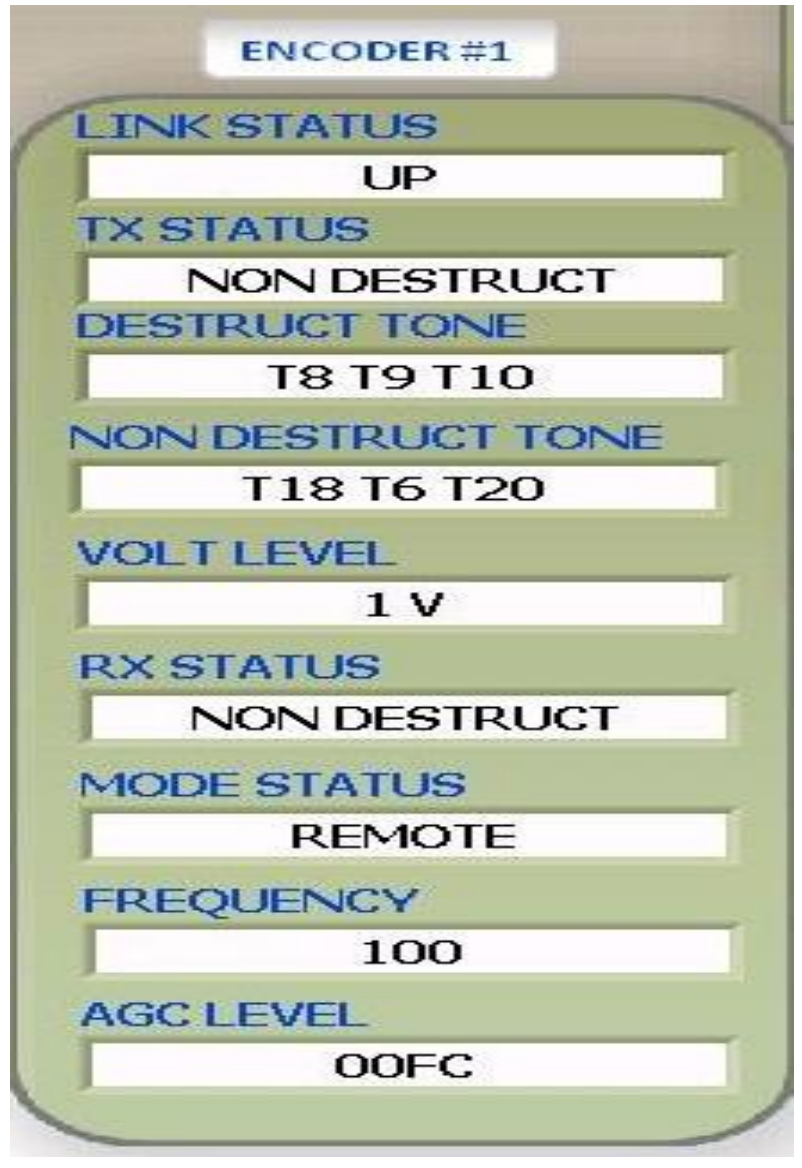

Figure 12. Displaying Encoder status information

Figure 12 represents the Encoder status information received through Ethernet port from Encoder change over unit. It displays the frequency used, tone used for different commands, transmitting status, receiving status and few other parameters.

\section{CONCLUSION}

The application developed here for monitoring and controlling of the baseband Encoder system is a total demonstration of Ethernet and RS 232 interface. The device is controlled i.e. commands are given through RS 232 port and the status of the device performance is monitored through Ethernet interface. Any such type of device having RS 232 control port and Ethernet interface can be interfaced to the application incorporating slight modification in the application.
This application has been tested and observed satisfactory result in recent past. The system performed satisfactorily for real-time monitoring and controlling.

The application can be placed in remote location and can be linked to the Encoder system through standard communication link and can be used for real time monitoring of Encoders parameter status and controlling.

\section{REFERENCES}

[1] LabVIEW 2009 by National Instruments, http://www.ni.com.

[2] LabVIEW Graphical Programming by Gary W johnsion (TMH).

[3] Zhou Hongfu; Xiao Xinyan; Tang Yong, "Serial Communication Interface Design Based on Lab VIEW and VC Mix Programming", 8th International Conference on Electronic Measurement \& Instruments, (ICEMI2007) PP44-49, (in English) (ISTP/EI, INSPEC).

[4] Machacek J.; Drapela J, "CONTROL OF SERIAL PORT (RS-232) COMMUNICATION IN Lab VIEW”, 9th International Conference Modern Technique and Technologies, (MTT 2008) PP36-40.

[5] Sun Jian; Lu Peng; Fu Yaqiong; Liu Ruixiang; Chen Le, "The Implementation and Application of Programming Port Communication between Industry PC and Mitsubishi FX Series PLC", 3rd International Conference Intelligent System and Knowledge Engineering-2008 (ISKE 2008), Volume: 1, PP 1324 1327.

[6] iao Lai; Huiqin Jia, "Design and implementation of Square-Cabin Environmental Parameters Monitoring System based on the virtual instrument", International Conference on Electronic and Mechanical Engineering and Information Technology (EMEIT-2011), Volume: 3, PP $1304-1307$.

[7] Svinov, A.; Turygin, Y.V.; Sitar, J, "Remote control system and its development for linear asynchronous motor", MECHATRONIKA, 2010, 13th International Symposium, PP $105-108$.

[8] Joan Marler and Frank Nezrick, 877,"Interferometer Digital Feedback and Control System", Fermi National Accelerator Laboratory, 1998, Internal Note 877-23. 